\title{
Fraturas intertrocantéricas fixadas com parafuso dinâmico de quadril: Ensaio clínico randomizado comparando a via lateral de Hardinge e o acesso minimamente invasivo*
}

\section{Fixation of Intertrochanteric Fractures with Dynamic Hip Screws: Randomized Controlled Trial Comparing the Hardinge and Minimally-invasive Approaches}

\author{
Eduardo Lima de Abreu ${ }^{1}$ Jonas Byk ${ }^{2}$ Fernando Westphal ${ }^{2}$ \\ ${ }^{1}$ Escola Superior de Ciências da Saúde, Universidade do Estado do \\ Amazonas (UEA), Boca do Acre, AM, Brasil \\ 2 Programa de Pós-Graduação em Cirurgia, Universidade Federal do \\ Endereço para correspondência Eduardo Abreu, MSc, Escola Superior \\ de Ciências da Saúde, Universidade do Estado do Amazonas, Avenida \\ Amazonas (PPGRACI-UFAM), Manaus, AM, Brasil \\ Carvalho Leal, 1.777, Cachoeirinha, Manaus, Amazonas, 69065001, \\ Brasil (e-mail: ed_manaus@yahoo.com.br).
}

Rev Bras Ortop 2022;57(1):150-158.

\begin{abstract}
Resumo
Objetivo Comparar as técnicas de osteossíntese de fraturas intertrocantéricas com o parafuso dinâmico de quadril (dynamic hip screw, DHS, em inglês) pelas vias de acesso de Hardinge e minimamente invasiva do quadril, avaliando o tempo cirúrgico, o grau de dor no pós-operatório imediato, a perda hematimétrica, e os aspectos funcionais de mobilidade ativa. Métodos Estudo clínico randomizado e duplo-cego, em que 66 pacientes com fratura intertrocantérica foram submetidos a osteossíntese com DHS. Os pacientes foram divididos em um grupo teste, submetidos ao acesso minimamente invasivo, e um

Palavras-chave

- fratura intertrocantérica

- procedimentos cirúrgicos minimamente invasivos

- osteossíntese

- dor pós-operatória

- ensaio clínico controle, em que a cirurgia foi realizada pela via de Hardinge.

Resultados Os pacientes submetidos ao tratamento pela via minimamente invasiva apresentaram um grau de dor pós-operatória inferior em comparação ao grupo tratado pela via lateral de Hardinge $(p<0,001)$, assim como menor perda hematimétrica $(p<0,001)$, menor tempo operatório $(p<0,001)$, e melhora nos testes de mobilidade ativa pós-operatória imediata $(p<0,05)$.

Conclusão $O$ estudo demonstrou a superioridade clínica nos parâmetros analisados da via de acesso minimamente invasiva em relação ao acesso de Hardinge para a fixação de fraturas intertrocantéricas, quando o DHS for a opção de osteossíntese escolhida. Nível de evidência I.
\end{abstract}

Trabalho desenvolvido na Universidade do Estado do Amazonas (UEA), Boca do Acre, AM, Brasil. recebido

07 de Maio de 2020

aceito

14 de Outubro de 2021
DOI https://doi.org/

$10.1055 / \mathrm{s}-0042-1742348$ ISSN $0102-3616$. (c) 2022. Sociedade Brasileira de Ortopedia e Traumatologia. All rights reserved.

This is an open access article published by Thieme under the terms of the Creative Commons Attribution-NonDerivative-NonCommercial-License, permitting copying and reproduction so long as the original work is given appropriate credit. Contents may not be used for commercial purposes, or adapted, remixed, transformed or built upon. (https://creativecommons.org/ licenses/by-nc-nd/4.0/)

Thieme Revinter Publicações Ltda., Rua do Matoso 170, Rio de Janeiro, RJ, CEP 20270-135, Brazil 
Abstract

\section{Keywords}

- intertrochanteric fracture

- minimally-invasive surgical procedures

- osteosynthesis

- pain, postoperative

- clinical trial
Objective To compare the techniques for the osteosynthesis of intertrochanteric fractures with dynamic hip screws (DHSs) through the Hardinge and minimally-invasive access routes of the hip, evaluating the operative time, the degree of pain in the immediate postoperative period, the hematimetric loss, and the functional aspects of active mobility.

Methods A randomized, double-blinded clinical trial in which 66 patients with intertrochanteric fractures were submitted to osteosynthesis by DHS. The patients were divided into a test group, submitted to the minimally-invasive access, and a control group, in whom the surgery was performed through the Hardinge route.

Results Patients submitted to the minimally-invasive treatment presented a lower degree of postoperative pain compared to the group treated by the Hardinge lateral route $(p<0.001)$, as well as lower hematimetric loss $(p<0.001)$, shorter operative time $(p<0.001)$, and improvement in immediate postoperative active mobility tests $(p<0.05)$.

Conclusion The study demonstrated the clinical superiority of the minimally-invasive access route parameters analyzed in relation to the Hardinge access for the fixation of intertrochanteric fractures when DHS is the choice osteosynthesis method.

Level of evidence I.

\section{Introdução}

A fratura intertrocantérica é uma entidade nosológica de tratamento idealmente cirúrgico, comum em pacientes idosos, e sua prevalência é crescente, graças ao aumento da expectativa de vida da população em todo o mundo. A perda importante de densidade de massa óssea, própria dessa população, favorece a ocorrência destas fraturas motivadas por traumas de baixo impacto, como as quedas da própria altura. $^{1}$

Ao longo do tempo, foram desenvolvidos diversos implantes para a fixação de fraturas intertrocantéricas, sendo eles divididos em dois grupos: os de fixação intramedular e os de estabilização extramedular, entre os quais se destaca o parafuso dinâmico de quasril (dynamic hip screw, DHS, em inglês). Nos dias atuais, a fixação dessas fraturas com hastes cefalomedulares, apesar de ter maior custo, tem sido a opção da maioria dos autores devido à sua baixa agressividade às partes moles, pois se utilizam vias de acesso minimamente invasivas para sua instalação, e principalmente por se apresentar como um método que confere melhor estabilidade. ${ }^{2} \mathrm{O}$ DHS, no entanto, ainda permanece uma excelente opção de fixação de fraturas intertrocantéricas. ${ }^{3-5}$

Quando o DHS é o implante escolhido para o tratamento, a via de acesso classicamente utilizada é a descrita em 1982 por Hardinge, ${ }^{6}$ que consiste em uma abordagem de $15 \mathrm{~cm}$ a $20 \mathrm{~cm}$ na face lateral da coxa, com secção longitudinal da fáscia lata e dissecção do terço posterior do vasto lateral. Em $2011,{ }^{7}$ no entanto, uma via de acesso minimamente invasiva foi proposta com o intuito de tornar o procedimento de fixação de fratura intertrocantérica menos agressivo.

O principal benefício que a técnica minimamente invasiva traz em relação à cirurgia tradicional é a diminuição do tempo cirúrgico e a menor taxa de sangramento, mas não há um consenso quanto à superioridade dessa técnica em termos da recuperação pós-operatória dos pacientes ${ }^{8-10}$

O objetivo principal deste estudo é analisar as fraturas intertrocantéricas fixadas comDHS, comparando as vias de acesso minimamente invasiva (osteossíntese minimamente invasiva com placa, minimally-invasive plate osteosynthesis, MIPO, em inglês) e a tradicional de Hardinge quanto ao índice álgico pós-operatório. $O$ objetivo secundário é analisar se há uma superioridade da primeira via em relação ao tempo cirúrgico, aos índices de perda sanguínea, e ao grau de mobilidade ativa no pós-operatório imediato (POI).

\section{Métodos}

Este é um ensaio clínico randomizado e duplo-cego - cadastrado no Registro Brasileiro de Ensaios Clínicos sob o protocolo 238492489-composto por pacientes idosos, vítimas de trauma de baixa energia no quadril, que apresentavam fratura intertrocantérica, sem demais lesões associadas, e que foram submetidos a fixação com DHS entre março e dezembro de 2018.

Foram incluídos no estudo pacientes brasileiros, com idade $\geq 60$ anos, de ambos os sexos, com fraturas intertrocantéricas em condições clínicas adequadas ao tratamento cirúrgico proposto, que autorizaram sua participação por meio da assinatura do Termo de Consentimento Livre e Esclarecido. Os critérios de exclusão foram: pacientes que se autodeclararam indígenas, portadores de fraturas intertrocantéricas com obliquidade do traço reversa, cujo tratamento com DHS não é recomendado, pacientes com fraturas intertrocantéricas patológicas por doença tumoral maligna ou benigna, casos em que a equipe de anestesia se recusou a realizar o protocolo de analgesia intraoperatória escolhido para o estudo, pacientes que necessitaram de 
hemotransfusão no transoperatório ou POI, aqueles considerados com nível cognitivo baixo para compreender os questionamentos da pesquisa, e pacientes que foram a óbito no POI.

O tamanho da amostra foi estimado em relação à proporção de idosos acometidos por fraturas intertrocantéricas encontrada em estudo realizado por Martini et al., ${ }^{11}$ que foi de $22,7 \%$, e ao total anual aproximado dessas cirurgias em pacientes com a referida doença realizadas no serviço em que foi desenvolvido o estudo, que é de 80 cirurgias. Estabeleceuse um intervalo de confiança de $95 \%$ e uma precisão de $4,95 \%$ (margem de erro), e estimou-se um tamanho amostral mínimo de 62 idosos.

A amostra foi composta inicialmente por 70 pacientes; desses, 66 foram distribuídos aleatoriamente em 2 grupos: 33 no grupo I, composto pelos pacientes submetidos a cirurgia pela via clássica de Hardinge, e 33 no grupo II, composto pelos pacientes submetidos a cirurgia pela via MIPO. Ao todo, 4 pacientes foram excluídos do estudo: 2 do grupo I ( $\mathrm{n}^{\circ} 14$, que foi a óbito no POI, e $\mathrm{n}^{\circ} 28$, que necessitou de hemotransfusão intraoperatória), e 2 do grupo II $\left(n^{\circ} 17\right.$ e $n^{\circ} 25$, ambos com baixa capacidade cognitiva no primeiro dia do pós-operatório, o que dificultou a avaliação precisa do grau de dor e dos testes de mobilidade) ( - Tabela 1, material suplementar, disponível apenas online).

O estudo foi submetido ao Comitê de Ética em Pesquisa, e recebeu o Certificado de Apresentação para Apreciação Ética (CAAE: 68335517.1.0000.0007), e seguiu as Normas Consolidadas dos Relatórios de Ensaios Clínicos (Consolidated Standard of Reporting Trials, CONSORT, em inglês) desde a seleção dos pacientes até sua análise final, conforme demonstra a - Figura 1.

A randomização dos grupos foi realizada por meio da ferramenta eletrônica encontrada no endereço randomization.com.

Foi elaborado um questionário com as variáveis pesquisadas neste estudo, composto pelo grau da dor no pré- e pósoperatórios (utilizando a Escala Visual de Faces de WongBaker), pelo grau de mobilidade ativa no POI, com a solicitação de elevação ativa do membro operado e avaliação da capacidade de o paciente sentar-se com auxílio no leito. 0 protocolo apresentava ainda as variáveis para quantificar, comparativamente, a perda hematimétrica em coletas préoperatórias de rotina e no primeiro dia de pós-operatório (hemoglobina e hematócrito). Mensurou-se também o tempo necessário, em cada um dos métodos, para a implantação do sistema DHS e para a sutura da ferida operatória (-Figura 2).
Na admissão, os pacientes foram interpelados para avaliação de dor pela escala de Wong-Baker por um examinador independente (o mesmo examinador escolhido para realizar a avaliação pós-operatória), sempre às 8 horas da manhã do primeiro dia de internação. Essa avaliação foi feita com o examinador apresentando ao paciente a escala de WongBaker, graduada de zero a 10 pontos. A prescrição préoperatória de analgesia era padrão, com Dipirona, na dose de $1 \mathrm{~g}$ sistemático a cada 6 horas, e de $100 \mathrm{mg}$ de cloridrato de tramadol por resgate.

Todos os pacientes avaliados foram operados pelo mesmo cirurgião, e a equipe de anestesiologia seguiu o mesmo protocolo de anestesia, com bloqueio raquianestésico com cloridrato de bupivacaína hiperbárica a $0,5 \%$, na dose aproximada de $10 \mathrm{mg}$, associado à $100 \mathrm{mg}$ de cloridrato de tramadol, administrado de maneira endovenosa, diluído em soro durante o transoperatório. O tempo cirúrgico foi mensurado em dois momentos: primeiramente, um que se iniciava com a via de acesso e terminava com o fim da osteossíntese, e outro, usado para síntese da ferida cirúrgica.

O curativo cirúrgico foi o mesmo para os pacientes dos 2 grupos, com gaze em rolo, de $20 \times 5 \mathrm{~cm}$, com 16 camadas fixadas com faixa de esparadrapo de $25 \times 10 \mathrm{~cm}$ cobrindo toda a extensão das vias de acesso, com o intuito de cegar o examinador e o paciente. A troca desse curativo foi realizada no primeiro dia de pós-operatório, após a anamnese do paciente, utilizando-se o protocolo da pesquisa.

A prescrição de analgesia pós-operatória foi mantida, com $100 \mathrm{mg}$ de cloridrato de tramadol injetados no subcutâneo como resgate, e com $1 \mathrm{~g}$ de dipirona por via endovenosa sistemática a cada 6 horas. No primeiro dia do pós-operatório, os pacientes foram reavaliados pela escala de de WongBaker, e arguidos sobre a solicitação de medicação opioide. Após a nova mensuração da dor, os pacientes foram submetidos a duas avaliações para determinar o grau de mobilidade ativa do quadril. Inicialmente, eram auxiliados a se sentar no leito e a permanecer nesta posição por até 10 segundos; posteriormente, eram orientados a elevar o membro operado, com o joelho estendido, até um ângulo de aproximadamente $45^{\circ}$.

Nova coleta de sangue foi realizada no primeiro dia do pós-operatório, para avaliação da alteração hematimétrica, e comparada com o exame colhido antes da cirurgia.

O desfecho primário do estudo foi a avaliação do grau de dor no pós-operatório de acordo com a escala de WongBaker, e o desfecho secundário foi a avaliação do grau de mobilidade pós-operatória do quadril.

Tabela 1 Análise do grau de dor pré e pós-operatórias dos pacientes por meio da Escala Visual de Wong-Baker

\begin{tabular}{|l|l|l|l|l|l|l|}
\hline Grupo & $\mathbf{n}$ & \multicolumn{4}{l|}{ Dor pré-operatória } & \multicolumn{2}{l|}{ Dor pós-operatória } & $p^{*}$ \\
\hline & & Média & Desvio padrão & Média & Desvio padrão & \\
\hline Hardinge & 33 & 5,15 & 2,15 & 7,03 & 1,90 & 0,001 \\
\hline MIPO & 33 & 6,30 & 1,38 & 3,33 & 2,31 & $<0,0001$ \\
\hline
\end{tabular}

Abreviatura: MIPO, minimally-invasive plate osteosynthesis (osteossíntese minimamente invasiva com placa).

Notas: *Valores de $p<0,05$ (5\%) foram considerados estatísticamente significativos. Teste $t$ de Student. 


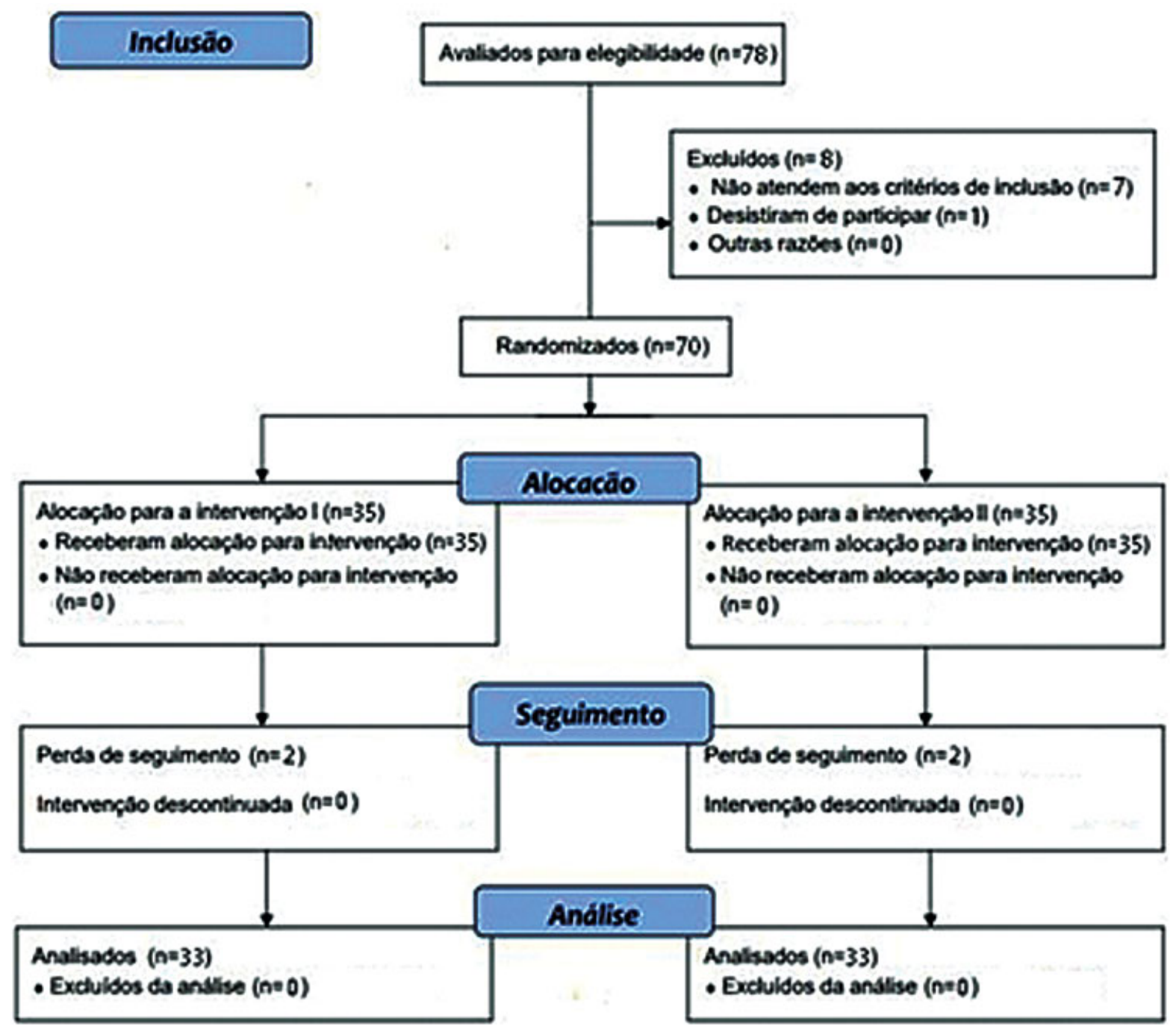

Fig. 1 Fluxograma dos estágios do ensaio clínico de idosos portadores de fratura intertrocantérica submetidos a fixação com DHS. Intervenção I: fixação pelo acesso de Hardinge; intervenção II: pela via minimamente invasiva.

\section{Resultados}

Dos 33 idosos do Grupo I, 15 (45,5\%) eram de sexo masculino, e 18 (54,5\%), do sexo feminino. No Grupo II, 19 (57,6\%) eram do sexo masculino, e $14(42,4 \%)$, do sexo feminino. A média de idade do grupo Ifoi de 80,42 $\pm 8,26$ anos, e a do grupo II, de 78,58 $\pm 8,78$ anos, e apresentou variação estatística aproximada entre os grupos $(p=0.191)$. A distribuição das fraturas com relação à classificação de Tronzo está demonstrada na - Figura 3.

Com relação à determinação do quadro álgico, o grupo I apresentou um aumento significativo do grau médio de dor, quando comparadas as avaliações pré- e o pós-operatórias (de 5,15 $\pm 2,15$ para $7,03 \pm 1,90$ ). Quanto aos pacientes do grupo II, o grau médio de dor no préoperatório foi de $6,30 \pm 1,38$, e no pós-operatório, de $3,33 \pm 2,31$, o que revela uma redução significativa $(p<0,0001)$ ( - Tabela 2, material suplementar, disponível apenas online). Quando comparadas as taxas de dor pósoperatória entre os grupos, a pontuação do grupo II foi significativamente menor do que a do grupo I $(p<0,0001)$, conforme mostra a - Tabela $\mathbf{1}$.
Ao comparar os níveis médios de hemoglobina e hematócrito dos pacientes no pré-operatório, observou-se que não havia diferença estatisticamente significativa entre os grupos I e II ( $p=0,643$ e $p=0,536$, respectivamente). No pósoperatório, a perda hematimétrica foi significativamente menor no grupo II ( - Tabela 2$)(p<0,001)$.

O tempo de cirurgia foi medido em duas etapas: o tempo para a osteossíntese, calculado do início da abertura da via de acesso até a colocação do último parafuso da placa, e o tempo necessário para o fechamento da ferida operatória. O tempo necessário para a realização da MIPO foi significativamente menor em ambas as etapas ( $p>0,001)$, como também mostra a - Tabela 2.

Após a avaliação da dor pós-operatória, foram realizados dois testes de mobilidade do quadril operado. No primeiro deles, solicitava-se ao paciente que se sentasse no leito e permanecesse nesta posição por 10 segundos. No segundo, pedia-se ao paciente que fletisse o quadril operado com o joelho estendido. Em ambos os testes, o grupo II obteve melhor desempenho $(p=0,031$ e $p=0,001$, respectivamente), conforme mostra a - Figura 4. 
154 Fraturas intertrocantéricas fixadas com parafuso dinâmico de quadril Abreu et al.

\section{Protocolo de Pesquisa}

Estudo Comparativo Entro a Via Lateral do Hardinge $\bullet$ o Acesso Minimamente Invasivo Para o Tratamento de Fraturas Intertrocantéricas, Com o Sistema Dynamic Hip Screw.

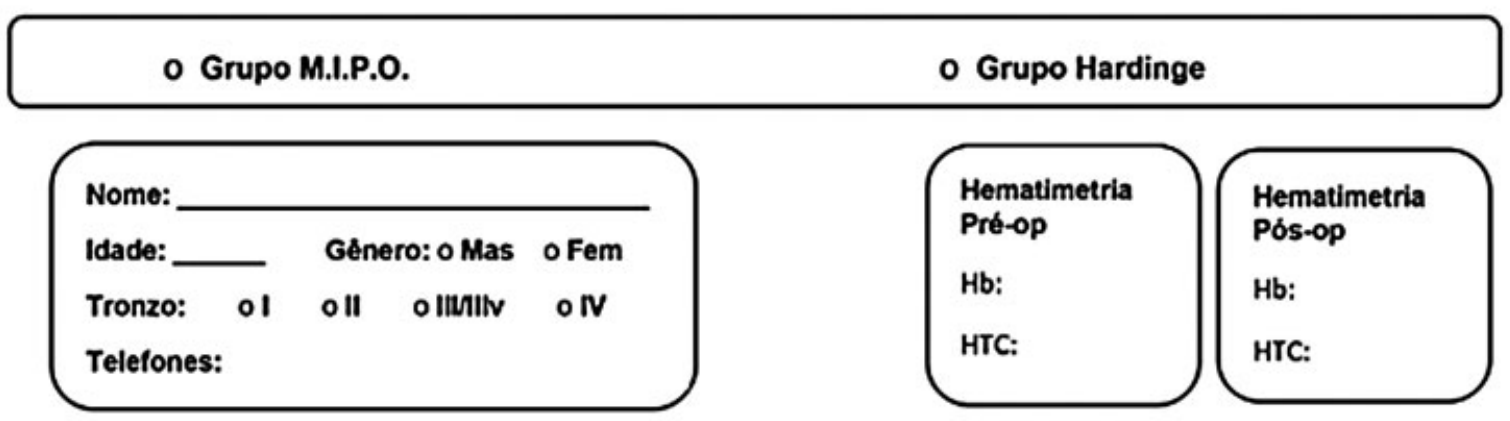

Escala de Wong \& Baker Pré-operatória

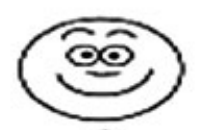

$\mathbf{0}$

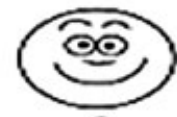

$\mathbf{0}$
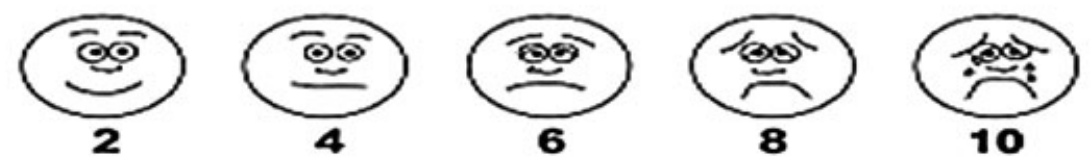

Escala de Wong \& Baker Pré-operatória
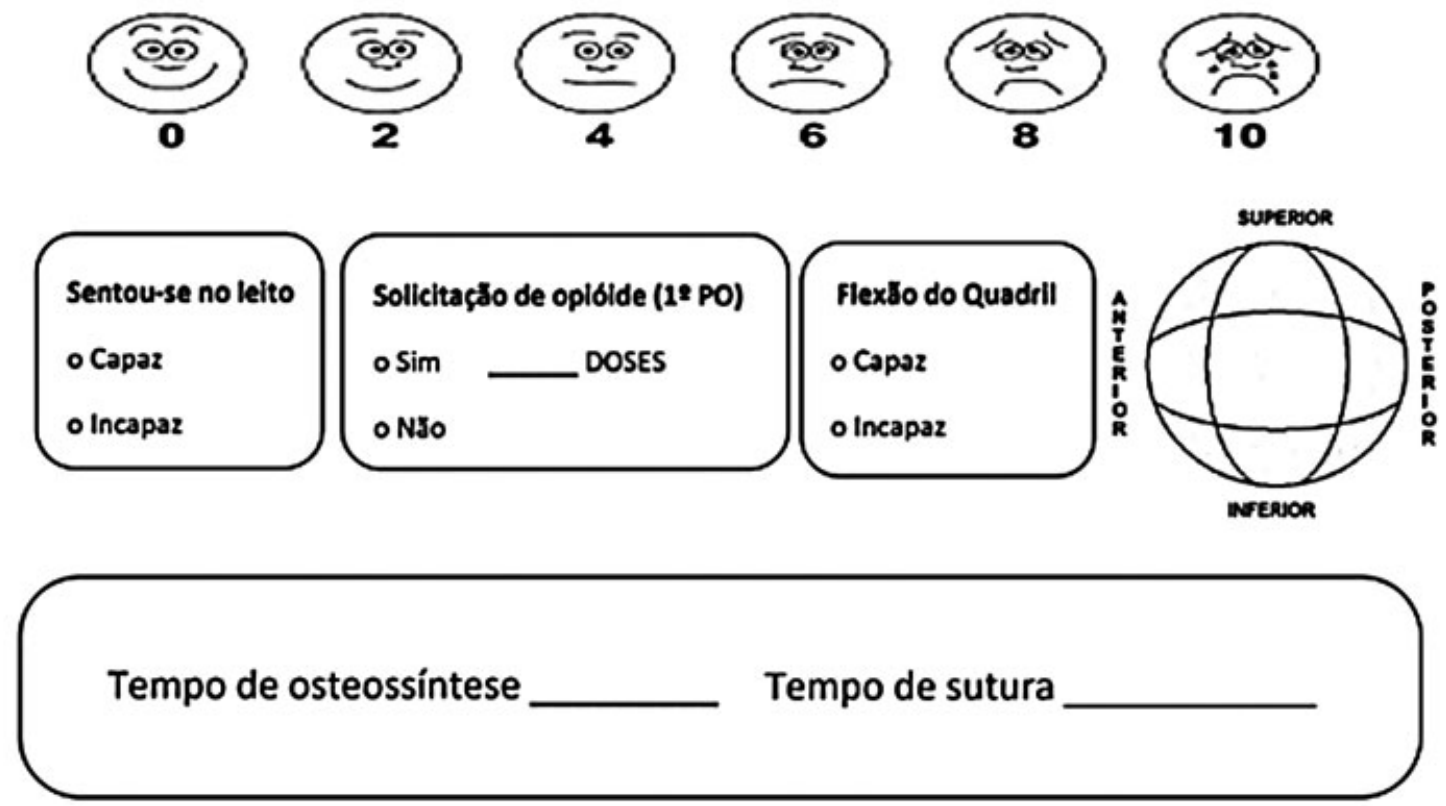

Fig. 2 Formulário para coleta de dados do protocolo de pesquisa.

Quanto ao posicionamento da ponta do DHS em relação aos quadrantes da cabeça femural, propostos pela Cleveland Femural Head Division, em 12 dos casos o DHS foi alocado no quadrante 4 , sendo $06(50,0 \%)$ do grupo I e $6(50,0 \%)$ do grupo II; 37 no quadrante 5 , sendo $20(54,1 \%)$ do grupo I e $17(45,9 \%)$ do grupo II; 12 no quadrante 6 , sendo $6(50,0 \%)$ do grupo I e $6(50,0 \%)$ do grupo II; 1 no quadrante 07 , que era do grupo II, e 4 idosos foram alocados no quadrante 8 , sendo $1(25,0 \%)$ do grupo I e $3(75,0 \%)$ do grupo II, como mostra a - Figura 5.

\section{Discussão}

O tratamento cirúrgico por MIPO em fraturas intertrocantéricas se mostrou superior, quando comparado ao tratamento pela via tradicional de Hardinge. Nos procedimentos em que a via de acesso foi menor, o grau de dor pós-operatória e a taxa de perda sanguínea foram menores, e a mobilização ativa no primeiro dia de cirurgia foi melhor.

As fraturas do quadril fazem parte de um universo extremamente amplo, e o conhecimento de sua abordagem é 


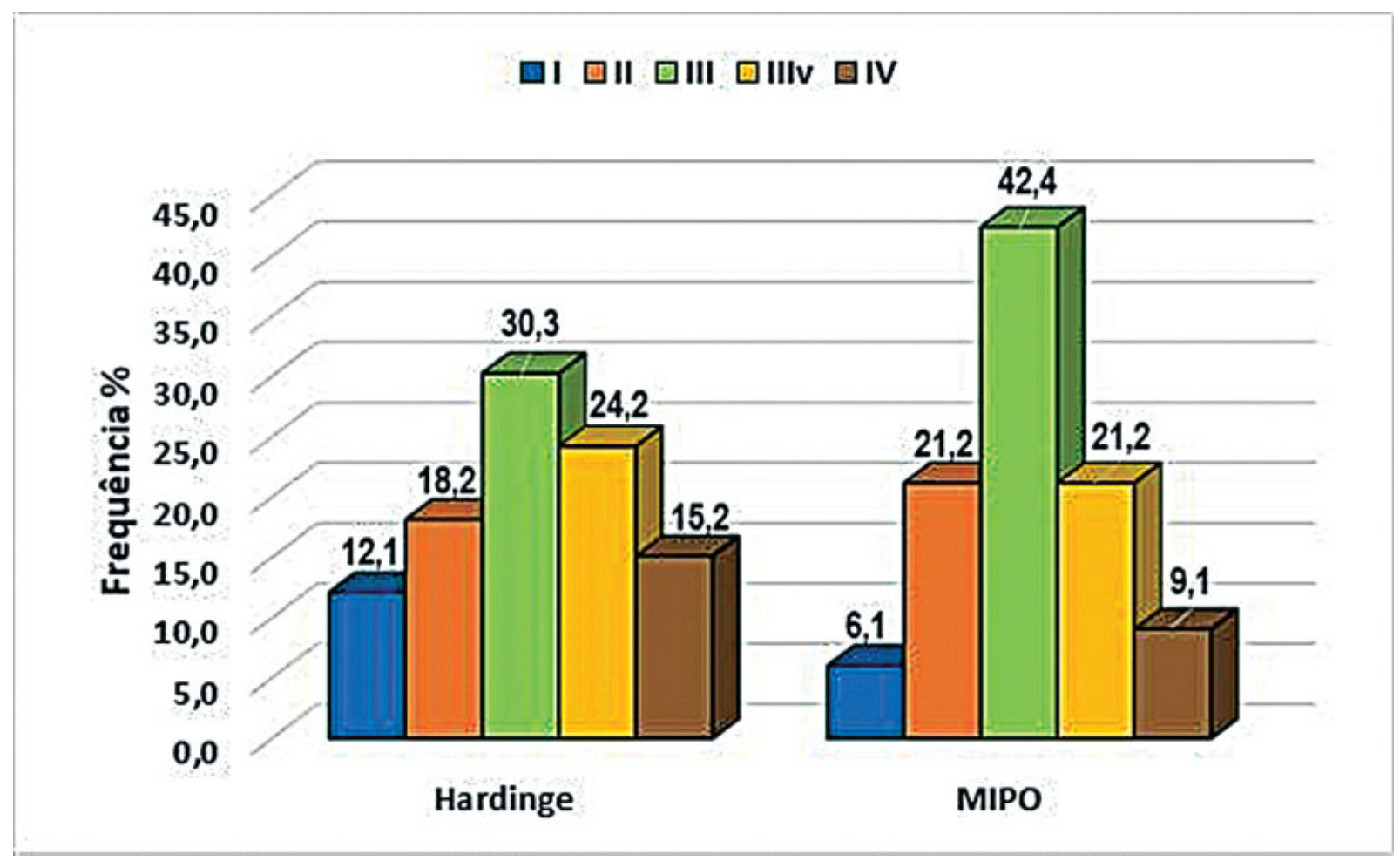

Fig. 3 Distribuição dos pacientes quanto ao tipo de fratura, de acordo com a classificação de Tronzo.

essencial, não apenas para o ortopedista ou cirurgião de quadril como para o médico de um modo geral. As fraturas intertrocantéricas, quando tratadas por MIPO, tiveram menor taxa de perda sanguínea, menor tempo cirúrgico, e resultaram em melhor recuperação funcional no POI. Por se tratar de uma condição peculiar em pacientes idosos e de tratamento ideaImente cirúrgico, essas fraturas apresentam uma morbidade elevada, e altas taxas de mortalidade. ${ }^{4,5,9,12,13}$

De modo geral, as cirurgias de trauma ortopédico evoluíram de uma era em que a redução anatômica com fixação rígida era preconizada. 0 problema desse princípio de osteossíntese era a notada abordagem direta da fratura, com importante agressão às partes moles e, invariavelmente, grande desperiostização dos fragmentos envolvidos, o que elevava o grau de agressão cirúrgica. A evolução das técnicas operatórias e o advento da fluoroscopia mostrou que uma abordagem menos agressiva, muitas vezes sem qualquer contato com o foco da fratura, tinha um índice bastante satisfatório de consolidação, e com menor agressividade cirúrgica. Começava aí a valorização das técnicas minimamente invasivas, com redução indireta das fraturas e fixação com máximo possível de preservação das partes moles adjacentes ao foco, com preservação do suprimento sanguíneo necessário para a cicatrização óssea. ${ }^{14}$

Seguindo a tendência mundial, o tratamento cirúrgico das fraturas intertrocantéricas também sofreu importantes alterações. Em 1982, o acesso de Hardinge, ${ }^{6}$ que foi descrito idealmente para realizar artroplastias do quadril, acabou por se popularizar no tratamento das fraturas intertrocantéricas. No entanto, essa abordagem ampla trazia consequências adversas ao paciente, principalmente com relação à perda sanguínea e ao grau de dor pós-operatória. ${ }^{7}$ A mudança no

Tabela 2 Comparação da perda hematimétrica e do tempo cirúrgico entre os grupos

\begin{tabular}{|c|c|c|c|c|c|}
\hline EXAMES & HARDII & & MIPO & & $p^{*}$ \\
\hline & Média & Desvio padrão & Média & Desvio padrão & \\
\hline Hemoglobina pré-operatória (mg/dL) & 12,62 & 0,85 & 12,52 & 0,90 & 0,643 \\
\hline Hematócrito pré-operatório $(\mathrm{mg} / \mathrm{dL})$ & 37,32 & 2,71 & 36,90 & 2,74 & 0,536 \\
\hline Hemoglobina pós-operatória (mg/dL) & 9,55 & 0,84 & 10,80 & 1,17 & $<0,0001$ \\
\hline Hematócrito pós-operatório (mg/dL) & 28,05 & 2,61 & 31,64 & 3,49 & $<0,0001$ \\
\hline Tempo de fixacão (minutos) & 46,55 & 5,92 & 21,03 & 5,09 & $<0,0001$ \\
\hline Tempo de sutura (minutos) & 14,27 & 3,56 & 6,15 & 1,48 & $<0,0001$ \\
\hline
\end{tabular}

Abreviatura: MIPO, minimally-invasive plate osteosynthesis (osteossíntese minimamente invasiva com placa).

Notas: *Valores de $p<0,05$ (5\%) foram considerados estatísticamente significativos. Teste $t$ de Student. 


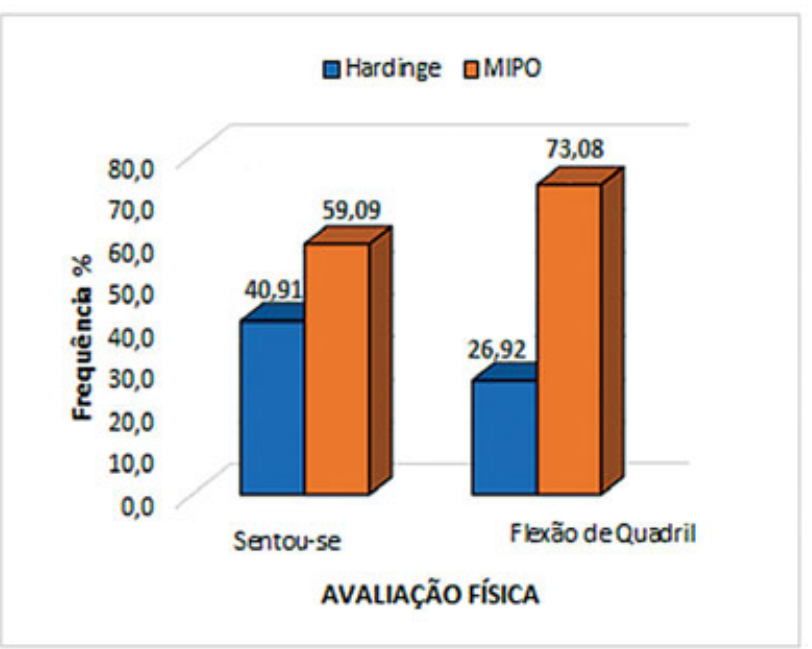

Fig. 4 Avaliação da mobilidade ativa pós-operatória por grupo.

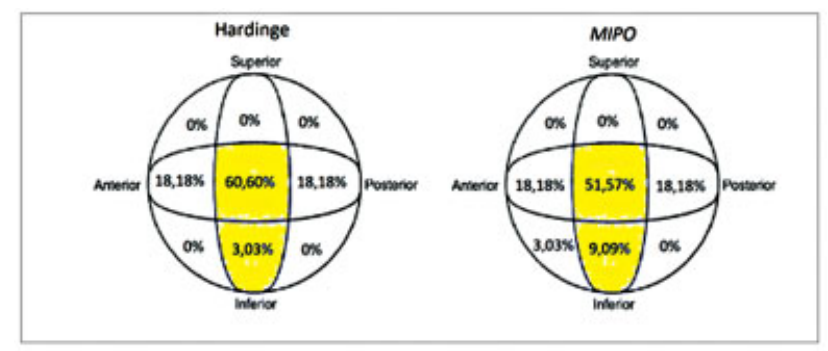

Fig. 5 Distribuição do posicionamento do ápice do parafuso deslizante em relação à cabeça femoral, de acordo com os quadrantes da Cleveland Femoral Head Division. No grupo I, o posicionamento foi considerado adequado em aproximadamente $63 \%$, e no grupo II, em aproximadamente $60 \%$.

manejo do tratamento dessas fraturas com o DHS se iniciou com um estudo ${ }^{7}$ com 40 pacientes, em que os autores concluíram que uma abordagem minimamente invasiva (MIPO) era segura, eficaz, reduzia a perda sanguínea operatória e o grau de dor, além de conseguir o alinhamento da redução e o posicionamento adequado do parafuso, que são fatores preditivos de bons resultados.

Os implantes extramedulares para a estabilização das fraturas intertrocantéricas também passaram por uma importante revolução, desde os modelos que não permitiam sua impacção, e consequentemente apresentavam elevados índices de falha mecânica, até o sistema desenvolvido por Richard com o parafuso deslizante, precursor do DHS, que revolucionou o tratamento desses pacientes. ${ }^{14}$

Em nota técnica publicada em $2012,{ }^{15}$ foi proposta a utilização do sistema MINUS, que era uma adaptação do DHS tradicional, que utilizava instrumentais próprios para realizar a cirurgia minimamente invasiva. A técnica consistia em instalar os implantes pela mini-incisão, sendo a fixação da placa à cortical do fêmur realizada de maneira percutânea, auxiliada por um gabarito adaptado ao sistema para orientar o bloqueio. ${ }^{15}$
No presente estudo, todas as cirurgias foram realizadas com o mesmo instrumental, sendo que, para as MIPOs, alguns tempos cirúrgicos foram readaptados, o que mostra que não é preciso nenhum instrumento especial para a realização adequada e segura deste procedimento pela via menor.

A opção de fixação da fratura intertrocantérica por um sistema intra- ou extramedular, como o DHS, é uma polêmica histórica. A maioria dos estudos ${ }^{1,3-5,16}$ defende o método intramedular para as fraturas intertrocantéricas mais instáveis. Em um estudo prospectivo com mais de 4 mil pacientes nos Estados Unidos, foi demonstrada a superioridade dos implantes intramedulares em comparação ao DHS tradicional, com relação ao tempo de internação após a cirurgia. De acordo com os autores, o menor tempo de internação dos pacientes submetidos ao procedimento com hastes minimiza os custos com esse tipo de implante, mais caro do que o DHS.

Em uma série de casos realizada no Brasil, ${ }^{14}$ o DHS - por meio de acesso minimamente invasivo - foi o método utilizado para o tratamento de fraturas intertrocantéricas. Os autores ${ }^{14}$ verificaram a consolidação em todos os 140 casos, com uma taxa de óbito de apenas $1,4 \%$ até o sexto mês de seguimento, o que mostra a eficiência do método como forma de estabilização da fratura e do acesso com menor agressividade para a melhora do índice de sobrevida dos pacientes.

Mahmood et al. ${ }^{9}$ compararam o acesso de Hardinge e o MIPO no tratamento de fraturas intertrocantéricas, e concluíram que ambas as técnicas são seguras, mas a instalação do DHS por MIPO demandou menor tempo cirúrgico, e resultou em menor volume de sangramento e menor tempo médio de internação.

O presente estudo avaliou apenas a técnica de fixação extramedular com o DHS no tratamento de fraturas intertrocantéricas. No entanto, os resultados obtidos demostram que a utilização deste implante por MIPO traz os benefícios no curto prazo encontrados na fixação com hastes: teoricamente, execução mais rápida e menos agressiva, e menor volume de sangramento. Neste estudo, a média de tempo tanto para a fixação das fraturas quanto para o fechamento da ferida operatória foi significativamente menor nos pacientes submetidos a MIPO.

Em estudo realizado em 2016, ${ }^{4}$ comparou-se o volume de sangramento entre as técnicas de fixação com haste femoral proximal antirrotação (proximal femoral nail antirotation, PFNA, em inglês) e com o DHS. Os autores ${ }^{4}$ concluíram que o sangramento visível era maior no grupo tratado com o DHS (em que o acesso cirúrgico para a instalação é maior), e, em contrapartida, o sangramento oculto era mais volumoso nos pacientes tratados com a haste (em que há sangramento intraósseo pela lesão do endósteo, causado pela instalação do implante). A soma das perdas perfez um total médio de $320 \mathrm{~mL}$ nos pacientes tratados com DHS, e de $368 \mathrm{~mL}$ nos tratados com o PFNA. ${ }^{4}$ Em uma revisão sistemática com metanálise, Huang et al. ${ }^{17}$ demonstraram não haver diferença estatisticamente significativa entre tais métodos quanto ao sangramento. 
No presente estudo, mensurou-se a perda hematimétrica por meio da medida do peso de hemoglobina por decilitro $(\mathrm{mg} / \mathrm{dL})$ no primeiro dia do pós-operatório. Os achados revelaram que os pacientes do grupo I apresentaram uma maior perda hematimétrica do que os do grupo II, o que comprova a maior agressividade às partes moles do acesso de Hardinger.

A avaliação da dor pós-operatória é uma tendência em todas as especialidades médicas. Na cirurgia de trauma ortopédico em particular, a mensuração da dor e a avaliação da qualidade de vida ganharam espaço na mesma proporção em que as cirurgias menos agressivas começaram a virar realidade. No presente estudo, a avaliação das pontuações álgicas pré- e pós-operatórias foi realizada com a escala Wong-Baker. Demonstrou-se que o grupo II apresentou um quadro doloroso com pontuação significativamente menor do que o grupo I.

Em um estudo de avaliação de dor pós-operatória em diferentes procedimentos cirúrgicos do quadril, foram comparadas várias cirurgias para o tratamento de fraturas dessa região. Observou-se que as fraturas intertrocantéricas tratadas com DHS e hastes cefalomedulares foram os procedimentos de maior índice álgico, e a sugestão dos autores foi a criação de novas alternativas de controle da dor e novos protocolos de reabilitação para essas fraturas.

Em um outro estudo, realizado na Bélgica, Heinrich et al. (2002) compararam o grau de dor pós-operatória em pacientes com fraturas intertrocantéricas fixadas com o DHS (pela via tradicional) e com placa percutânea de compressão. Neste estudo, os pesquisadores chegaram à conclusão de que as cirurgias percutâneas com placa de compressão evoluíram com menor dor e confirmaram a importância do mínimo acesso para as cirurgias do quadril. Além do menor grau de dor, as cirurgias com menor invasão demandaram menor tempo transoperatório. Esses resultados coincidem com os observados no presente estudo, em que os pacientes submetidos a MIPO demonstraram menores índices álgicos.

O presente estudo também demonstrou que o grau de movimentação ativa dos pacientes no primeiro dia do pósoperatório foi satisfatório, avaliado por meio de dois testes. Tanto no primeiro deles, em que o paciente era orientado a se sentar-se no leito com leve ajuda do examinador e manter-se nessa posição por até 10 segundos, quanto no segundo, em que solicitava-se ao paciente que fletisse o quadril operado com extensão do joelho até aproximadamente $45^{\circ}$ de elevação, o grupo II teve desempenho melhor.

Quanto ao posicionamento do parafuso deslizante na cabeça femoral, Cleveland et al. $^{20}$ descreveram um sistema em que a cabeça femoral era dividida em 9 zonas, sendo as zonas 1 (anterossuperior), 2 (centro-superior) e 3 (posterossuperior) as de maior risco de falha de síntese, e as zonas 5 (central) e 8 (centro-inferior), as de instalação mais recomendável do parafuso. A técnica de cirurgia com acesso minimamente invasivo - apesar de limitar o acesso à face lateral do fêmur proximal e, por consequência, dificultar a manipulação de instrumentais do sistema de fixação - não pareceu um obstáculo ao posicionamento do parafuso deslizante em seu local mais ideal. As zonas 5 e 8 foram atingidas em mais de $60 \%$ dos casos, tanto nos pacientes submetidos à fixação pelo método tradicional, quanto pela via minimamente invasiva. Esse fato, associado ao menor tempo cirúrgico conseguido no grupo da cirurgia menos invasiva comprova que este acesso traz benefícios ao paciente e não oferece dificuldade técnica ao procedimento.

Entre as limitações deste estudo, destaca-se o fato de o seguimento ter sido muito curto, apenas no primeiro dia do pós-operatório. Devido a isso, as principais complicações de cirurgias de fixação do quadril em pacientes idosos, como infecções sistêmicas e do sítio cirúrgico, tromboses venosas com ou sem eventos tromboembólicos, falhas de síntese e óbito em qualquer etapa do seguimento que não seja no POI, não foram investigadas.

\section{Conclusão}

A via de acesso minimamente agressiva para o tratamento de fraturas intertrocantéricas apresentou melhores desfechos clínicos e funcionais do que a via de Hardinge em relação ao tempo cirúrgico, perda sanguínea, e grau de dor pós-operatória imediata.

\section{Fontes De Financiamento}

Esta pesquisa não recebeu nenhuma bolsa específica de agências de fomento nos setores público, comercial, ou sem fins lucrativos.

Conflito de Interesses

Os autores declaram não haver conflito de interesses.

\section{Referências}

1 De Abreu EL, de Oliveira MH. Avaliação da qualidade de vida dos pacientes submetidos à hemiartroplastia do quadril. Rev Bras Ortop 2015;50(05):530-536

2 Sahin S, Ertürer E, Oztürk I, Toker S, Seçkin F, Akman S. Radiographic and functional results of osteosynthesis using the proximal femoral nail antirotation (PFNA) in the treatment of unstable intertrochanteric femoral fractures. Acta Orthop Traumatol Turc 2010;44(02):127-134

3 Sambandam SN, Chandrasekharan J, Mounasamy V, Mauffrey C. Intertrochanteric fractures: a review of fixation methods. Eur J Orthop Surg Traumatol 2016;26(04):339-353

4 Yu W, Zhang X, Zhu X, et al. Proximal femoral nails anti-rotation versus dynamic hip screws for treatment of stable intertrochanteric femur fractures: an outcome analyses with a minimum 4 years of follow-up. BMC Musculoskelet Disord 2016;17:222

5 Egol K. Intertrochanteric hip fractures: the sliding hip screw. In: Wiss D. Master techniques in orthopaedic surgery: fractures. Philadelphia: Lippincott Williams \& Wilkins; 2013:331-341

6 Hardinge K. The direct lateral approach to the hip. J Bone Joint Surg Br 1982;64(01):17-19

7 Agrawal M, Yuvarajan P, Maini L. Modified minimally invasive approach for dynamic hip screw fixation. Eur J Orthop Surg Traumatol 2011;21(05):375-379

8 Vidyarthi K, Paluvadi S, Sinha A. Minimally invasive dynamic hip screw for intertrochanteric fractures: comparison with conventional method and surgical tips. Int J Res Orthop 2017;3(04): 712-717 
9 Mahmood A, Kalra M, Patralekh MK. Comparison between Conventional and Minimally Invasive Dynamic Hip Screws for Fixation of Intertrochanteric Fractures of the Femur. ISRN Orthop 2013;2013:484289

10 Ropars M, Mitton D, Skalli W. Minimally invasive screw plates for surgery of unstable intertrochanteric femoral fractures: a biomechanical comparative study. Clin Biomech (Bristol, Avon) 2008;23 (08):1012-1017

11 Martini LA, Moura EC, Santos LC, Malta DC, Pinheiro MdeM. Prevalence of self-reported diagnosis of osteoporosis in Brazil, 2006. Rev Saude Publica 2009;43(Suppl 2):107-116

12 Ho M, Garau G, Walley G, et al. Minimally invasive dynamic hip screw for fixation of hip fractures. Int Orthop 2009;33(02): 555-560

13 Kammerlander C, Neuerburg C, Verlaan JJ, Schmoelz W, Miclau T, Larsson $S$. The use of augmentation techniques in osteoporotic fracture fixation. Injury 2016;47(Suppl 2):S36-S43

14 De Abreu EL, Sena CB, Saldanha Rodrigues Filho as. Effectiveness of treatment of transtrochanteric fractures with Dynamic Hip Screws using minimally invasive access. Rev Bras Ortop 2016;51 (02):138-142
15 Sawaia RN, Belangero WD. THE MINUS(®) System Minimally Invasive Technique For The Treatment Of Trantrochanteric Fractures. Rev Bras Ortop 2012;47(01):113-117

16 Zhang K, Zhang S, Yang J, et al. Proximal femoral nail vs. dynamic hip screw in treatment of intertrochanteric fractures: a metaanalysis. Med Sci Monit 2014;20:1628-1633

17 Huang X, Leung F, Xiang Z, et al. Proximal femoral nail versus dynamic hip screw fixation for trochanteric fractures: a meta-analysis of randomized controlled trials. ScientificWorldJournal 2013;2013:805805

18 Foss NB, Kristensen MT, Jensen PS, Palm H, Krasheninnikoff M, Kehlet $H$. The effects of liberal versus restrictive transfusion thresholds on ambulation after hip fracture surgery. Transfusion 2009;49(02):227-234

19 Janzing HM, Houben BJ, Brandt SE, et al. The Gotfried PerCutaneous Compression Plate versus the Dynamic Hip Screw in the treatment of pertrochanteric hip fractures: minimal invasive treatment reduces operative time and postoperative pain. J Trauma 2002;52(02):293-298

20 Cleveland M, Bosworth DM, Thompson FR, Wilson HJ Jr, Ishizuka T. A ten-year analysis of intertrochanteric fractures of the femur. J Bone Joint Surg Am 1959;41-A:1399-1408 